\title{
Mesure des concentrations et des vitesses dans un courant mixte d'air et d'eau
}

\section{The measurement of concentrations and of velocities in a current of air and water mixed}

\author{
PAP G. HALBRONN \\ Ingéneur des ponts at Chausstés \\ English synopsis, p. 354 .
}

\section{Nature du problème}

Dans le domaine des écoulements uniformes at surface libre, l'attention des hydrauliciens s'est portée principalement, jusqu'à ce jour, sur létude des courants de pente modérée. Dans ce cas, il existe toujours une surface nette de séparation entre l'air, milieu ambiant, et le liquide; au contraire, lorsque la pente est forte et que de grandes vitesses sont atteintes, lobservation montre qu'il peut ne pas en etre ainsi : l'agitation turbulente devient telle qu'il n'existe plus de surface délimitant les domaines de la phase gazeuse et de la phase liquide. Tandis que des gouttelettes liquides sont projetées à de grandes hauteurs, des bulles gazeuses pénètrent dans le liquide et s'écoulent avec lui, produisant un aspect caractéristique d' " eau blanche ».

Les lois de l'écoulement du fluide homogène et les lois de l'écoulement du mélange sont complètement différentes, mais très peu d'études expérimentales ont été consacrées, jusqu'ici, à la rccherche de ces dernieres. Ce fait est imputable à l'absence de moyens de mesure appropriés; nous nous proposons de relater les travaux poursuivis au Laboratoire Dauphinois d'Hydraulique, afin de combler cette lacune.

Contrastant avec le cas d'un écoulement hom mogène défini par la mesure de la vitesse en chaque point, la description complète d'un écoulement mixte nécessite la connaissance locale de plusieurs grandeurs, a savoir :

$1^{\circ}$ La concentration spatiale moyenne en air $c_{s}$ définie comme étant la moyenne dans le temps du volume d'air contenu dans un volume unite entourant le point considéré. La concentration spatiale moyenne en eau est alors :

$$
\overline{\gamma_{s}}=1-\overline{c_{s}}
$$

$2^{\circ}$ I La vitesse moyenne de l'eau $\bar{v}_{e}$. Si $d Q_{\text {e }}$ re- présente le débit d'eau traversant à un instant donné une petite surface do centrée au point considéré et normale à la direction de l'écoulement, la vitesse instantanće de l'eau $v_{e}$ est définie par l'egalité :

$$
d Q_{c}=\gamma_{s} v_{c} d s
$$

Si les fluctuations de débit et de concentration sont assez faibles, on pourra écrire :

$$
\overrightarrow{d Q}=\psi_{s} \overline{v_{c}} d s
$$

qui définit $\ddot{v}_{c}$.

3" Eventuellement, la vitesse moyenne de l'air $\overrightarrow{b_{n}}$, donnée de mème par :

$$
\overrightarrow{d Q_{a}}=\bar{c}_{s} \bar{v}_{a} d s
$$

où $d Q_{a}$ représente le débit d'air moyen traversant $d_{\sigma}$. Si $v_{a}$ est différent de $v_{c}$, nous définissons la vitesse moyenne de glissement $\bar{v}_{i}$ par

$$
v_{l}=\overrightarrow{v_{t}}-\overrightarrow{v_{c}}
$$

L'expérience acquise dans le domaine de la mesure des concentrations spatiales des matériaux transportés en suspension nous a incite, pour déterminer $\overline{c_{s}}$, à utiliser des sondes reliant la résistivité électrique du mélange air-eau à sa composition. Nous nous proposons de décrire les premiers types de sondes réalisés, les inconvénients qu'ils présentaient et la sonde bifilaire qui nous a permis de venir à bout des difficultés rencontrées. Nous exposerons également la solution adoptée pour la mesure des vitesses.

Afin de mettre au point ces différents instruments, il a été nécessaire d'élablir une installation spéciale d'essais oì nous espérions créer une mixture de composition connue; quoique les expériences ultérieures aient montré que nous n'y étions pas parvenus, cette installation nous a 
cependant permis (par une méthode différente de celle qui était prévue initialement), de conclure à un fonctionnement correct des sondes réalisées.

\section{II. - Description de l'installation d'essai}

1) PRINCIPE DE LA CONFECTION D'UN MÉLANGE DE COMPOSITYON CONNUE

Nous avons défini ci-dessus les concentrations spatiales $\overline{c_{s}}$ et $\gamma_{s}$; mais les quantités directemenl mesurables a l'aide des instruments habituels sont : le débit global en cau $\bar{Q}_{\text {o }}$ et le débit global en air $Q_{k}$. On peut définir les concentrations de transport en air et en cau par les égatités:

$$
\begin{gathered}
\mathrm{C}_{\mathrm{C}}=-\frac{\overline{\mathrm{Q}_{n}}}{\overline{\mathrm{Q}_{c}}+\overline{\mathrm{Q}_{a}}} \\
\mathrm{r}_{\mathrm{T}}=\frac{\overline{\mathrm{Q}_{c}}}{\overline{\mathrm{Q}_{c}+\overline{\mathrm{Q}}_{t}}}=1-\mathrm{C}_{\mathrm{T}}
\end{gathered}
$$

Soit z la section de l'écoulement, on a :

(1) $\overrightarrow{Q_{w}}=\iint_{\Sigma} \vec{\gamma}_{s} \bar{v}_{v} d \sigma=\iint_{v}\left(1-\bar{c}_{s}\right) \bar{v}_{e} d \sigma$

De nìme :

(2) $\bar{Q}_{a}=\iint_{Y} \bar{c}_{s} \vec{v}_{d} d \sigma=\iint_{Y} \bar{c}_{w}\left(\vec{v}_{c}+\vec{v}_{g}\right) d \sigma$

Supposons que $\bar{v}_{\|}$soit négligeable devant $\bar{v}_{t}$. Alors :

$$
\mathrm{C}_{T}=\frac{\iint_{\Sigma} \overline{c_{N}} \overline{v_{c}} d \sigma}{\iint_{2} \overline{v_{c}} d \sigma}
$$

La concentration des transports n'est liee die facon simple à la concentration spatiale que si $\overrightarrow{c_{s}}$ ou $\overrightarrow{v_{e}}$ sont constants dans la section $\Sigma$; dans les deux cas :

$$
\mathrm{C}_{\mathrm{T}}=\frac{1}{\mathrm{~S}} \iint_{\mathrm{S}} \overline{c_{s}} d \sigma=\overline{\mathrm{C}}_{\mathrm{S}}
$$

Nous avons done tenté de réaliser une section de mesure où la vitesse soit uniforme et le glissement faible. De la mesure de $\bar{Q}_{c}$ et $\bar{Q}_{a}$, on au- rait alors deduit $\mathrm{C}_{\mathrm{r}}$ qui devait êtıe identique à la valeur moyenne spatiale $\overline{\mathrm{C}_{s}}$ de $\overline{c_{s}}$ mesuré en chaque point à la sonde électrique.

\section{2) Réalisation pratigue}

L'inslallation est verticale afin d'annuler le gradient transversal de $c_{s}$ dû̀ à la pesanteur. Une pompe Bergeron AK 34 refoule un débit maximum de $65 \mathrm{l} / \mathrm{s}$ dans une conduite de $350 \mathrm{~mm}$ de diamètre, prolongée par un convergent 350-150 d'un tracé tel qu'il donnerait une plage de vitesse uniforme à $1,5 \%$ près en eau pure (1). La section de mesure (fig. 1) est placée au col du con-

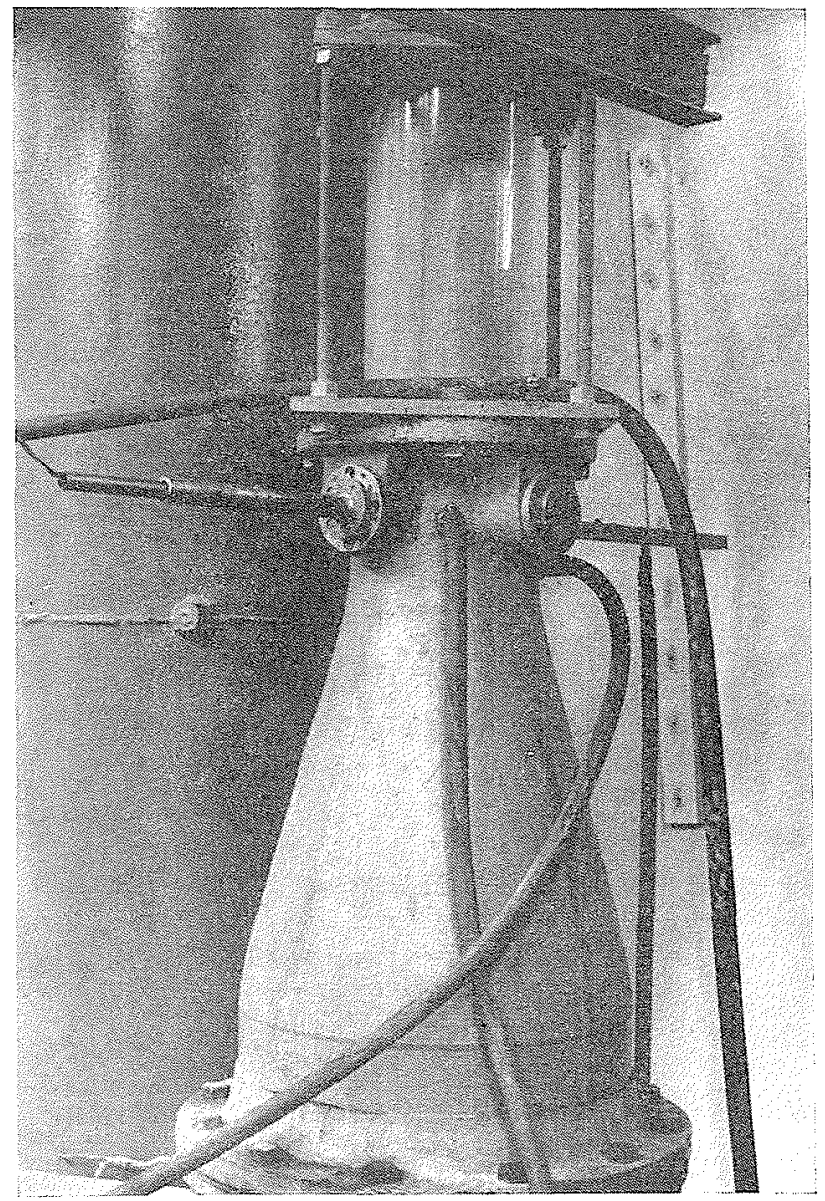

Fig, 1, - Secton de misure,

vergent : les instruments d'essai peuvent y ètre introduits à travers l'un ou l'autre de deux bossages formant étanchéité disposés sur deux axes rectangulaires. Un troneon de conduite en plexiglass permet d'observer l'écoulement immédiate-

(1) P. Lenoux et R. Bourobat. - Compte rendu des travaux de la S.H.F., n" 38,28 aveil 1928 , p. $387 \mathrm{H}$. 
ment à laval de la section de mesure (fig. 2). A la partic supérienre du modèle, la conduite débouche dans un bassin de dégazage suivi d'un déversoir triangulaire a $90^{\circ}$ pour la mesure du débit liquide. L'eau fait ensuite retour au hassin d'aspiration de la pompe.

L'air, fourni par un compresseur volumetrique, est introduit à la partie inférieure de la conduite de 350 , a travers me rampe réalisant une distribution aussi uniforme que possible de la concentration.

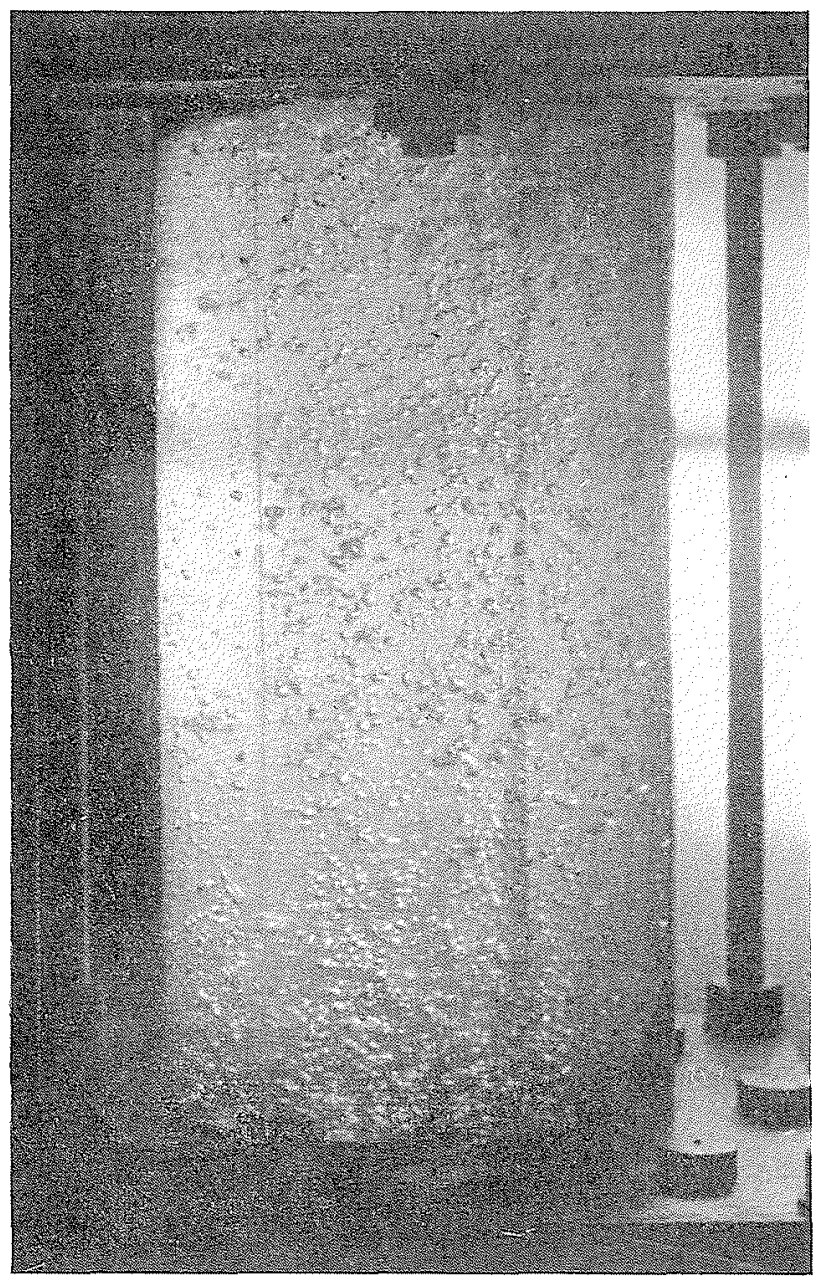

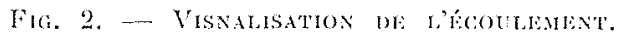

La mesure du débil gazenx dans les conditions normales est assuréc au moyen d'un débitmitre à diaphragme conforme à la norme AFNOR $\mathrm{x}$ 10-101.

Le diaphragme le plus couramment utilisé avait un diametre de $20 \mathrm{~mm}$.

Pour connaître le débit volumétrique de l'air dans la section de mesure, fonction des conditions locales de pression el de température, on détermine en outre :
- la pression statique au col du convergent, au moyen d'une prise de la paroi reliée à un manomètre à mercure;

- la température de l'eau, en équilibre thermique avec l'air, au moyen d'un thermomitre à mercure au demi-degré.

Pour un débit total de $80 \mathrm{~J} / \mathrm{s}$, la vitesse moyenne dans la section de mesure est de $4,50 \mathrm{~m}$. Certaines expériences (2) ayant montré que, pour des bulles isolées, la vitesse ascensionnelle croît en fonction du rayon de ces bulles pour atteindre une valeur limite de $0,24 \mathrm{~m} / \mathrm{s}$ environ, lorsqu'il dépasse $2 \mathrm{~mm}$, nous pensions que le rapport $\frac{\bar{v}_{g}}{\bar{v}_{c}} \# \frac{0,24}{4,5}=5,3 \%$ demeurerait faible et que ce fait, joint à l'uniformité de la plage des vitesses, devait entraîner l'identité des valeurs de $\mathrm{C}_{\mathrm{t}}$ mesuré directement et de $\overline{\mathrm{C}_{\mathrm{s}}}$ mesuré à la sonde électrique. L'expérience ne devait pas confirmer cette prévision.

\section{III. - Dispositif électrique de mesure}

Le principe des mesures consiste ì comparer la résistance $R_{0}$ d'une sonde lorsqu'elle est immergée dans l'eau pure et sa résistance R lorsqu'elle est immergée dans la mixture. Liensemble du montag̣e électrique est décrit dans l'article de M. Bounan. Une des difficultés de la méthode réside dans la variation possible de $R_{0}$ au cours de la mesure; en particulier, la résistivité de l'eau rariant de $3 \%$ par degré, le moindre changement de température a une grosse influence. Dans le cas présent, le tableau I montre que, en dépit de la température assez clevée de l'air injecté, la température dans la conduite ne varie pratiquement pas, grâce au grand volume du bassin d'alimentation el a la forte capaeité calorifique de l'eau devant celle de l'air.

TABLEAU I

\begin{tabular}{|r|c|c|c|c|c|}
\hline Temps, minutes...... & 0 & 7 & 10 & 15 & 20 \\
Température de l'air in- & & & & & \\
jecté, degrés........ & 15 & 18 & 21 & 25 & 30 \\
Temper. de l'eau, degrés & 12 & 12 & 12 & 12, & 12,1 \\
\hline
\end{tabular}

Datilleurs, la mesure de $R_{0}$ avanl et apres chaque mesure de $R$ aurait permis de déceler une variation s'il s'en ctail produit une.

(2) Mrracir. - Phil. Mag. Vol. 50, n"295, juillet 1925, p. 112. 


\section{IV. - Essais de sondes à plaques}

\section{1) PRInCIPE.}

L'air se comportant comme un isolant vis-àvis de l'eau, la résistance d'un certain volume de mixture est supérieure à celle du même volume d'eau pure. Pour des particules d'isolant sphériques disposées régulièrement dans un entrefer cubique de coté $l$, Lord RAYlergh (3) a montré que :

$$
\left.\frac{1}{\mathrm{R}}=\frac{l}{\mathrm{\rho}} \mid 1-\frac{3 c_{s}}{2+c_{s}-0,98 c_{s}{ }^{10 / 3}+\ldots}\right\rceil
$$

q désignant la résistivité du corps conducteur. Alors :

$$
\frac{1}{\mathrm{R}_{0}}=\frac{l}{?}
$$

et :

$$
c_{s}=\frac{2}{3} \frac{\mathrm{R}-\mathrm{R}_{0}}{\mathrm{R}}\left[1+\frac{1}{3} \frac{\mathrm{R}-\mathrm{R}_{0}}{\mathrm{R}}+\cdots\right]
$$

Pour les faibles concentrations, on obtient donc la formule simple :

$$
c_{s}=\frac{2}{3} \frac{\mathrm{R}-\mathrm{R}_{\mathrm{n}}}{\mathrm{R}}
$$

Le terme quadratique représente déjà $10 \%$ du terme principal lorsque $c_{s}=0,2$. Il est nécessaire d'en tenir compte pour des concentrations supérieures, ce qui complique considérablement la prise de la moyenne par l'appareillage électrique.

La validité de la formule (3) a été vérifiée expérimentalement au laboratoire; elle fournit un moyen de mesurer les concentrations en air lorsque les bulles sont effectivement sphériques, de taille uniforme et régulièrement distribuées.

\section{2) Ríalisation.}

La sonde $\mathrm{n}^{\circ} 1$ (fig. 3 ) comportait deux électrodes en acier inoxydable de $20 \times 20 \times 1$, distantes de $20 \mathrm{~mm}$ et montées sur des plaques de plexiglass de $2 \mathrm{~mm}$ d'épaisseur reliées par un culot également en plexiglass abritant la sortie des fils de mesure; l'ensemble était supporté par un tube de laiton 12.16 .

Les premiers essais révélèrent un certain nombre d'inconvénients :

a) Le champ n'est pas limité à sa partie uni-

(3) Lord Rayleigh. - On the influence of obstacles arranged in rectangular order upon the properties of $a$ medium. Scientific Papers, Vol. IV, n² 200, p. 19. forme entre les électrodes, mais comporte des " anses de panier" importantes à l'extérieur. Ce fait est mis en particulier en évidence par la variation de $\mathrm{R}_{0}$ lorsqu'on approche la sonde d'une paroi de la conduite. La figure 4 montre une variation de 5,5/1.000 de $R_{0}$ lorsque le centre de la sonde se trouve à $15 \mathrm{~mm}$ de la paroi; dans ces

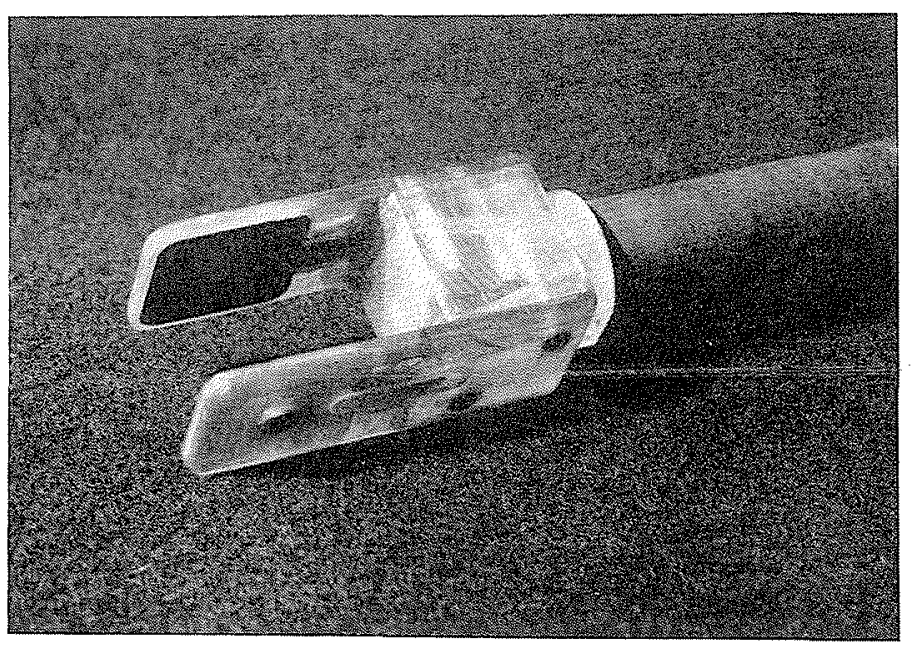

Fia. 3. - Sonde $\mathrm{N}^{\mathrm{a}} 1$.

conditions, il serait impossible de mesurer la concentration en air au voisinage du fond d'un canal.

De plus, la formule (3) est valable pour un champ cubique, mais non pour un champ comportant des anses de panier. Enfin, la sonde pla-

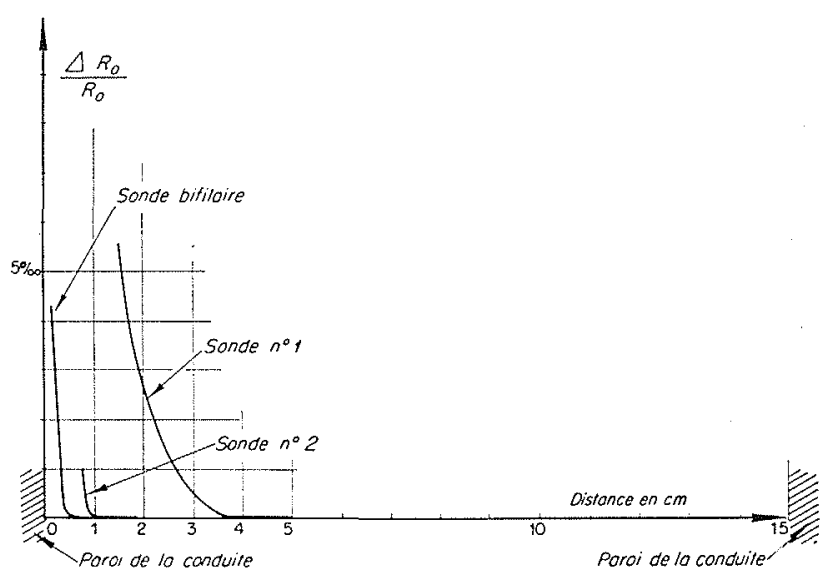

FIG. 4.

cée dans une mixture présentant un gradient de concentration ne donne pas une indication liée de manière simple à la valeur de $c_{s}$ en son centre.

Afin de remédier à ces inconvénients, nous avons réalisé une sonde comportant des plaques de garde, mais ses dimensions étaient prohibi- 


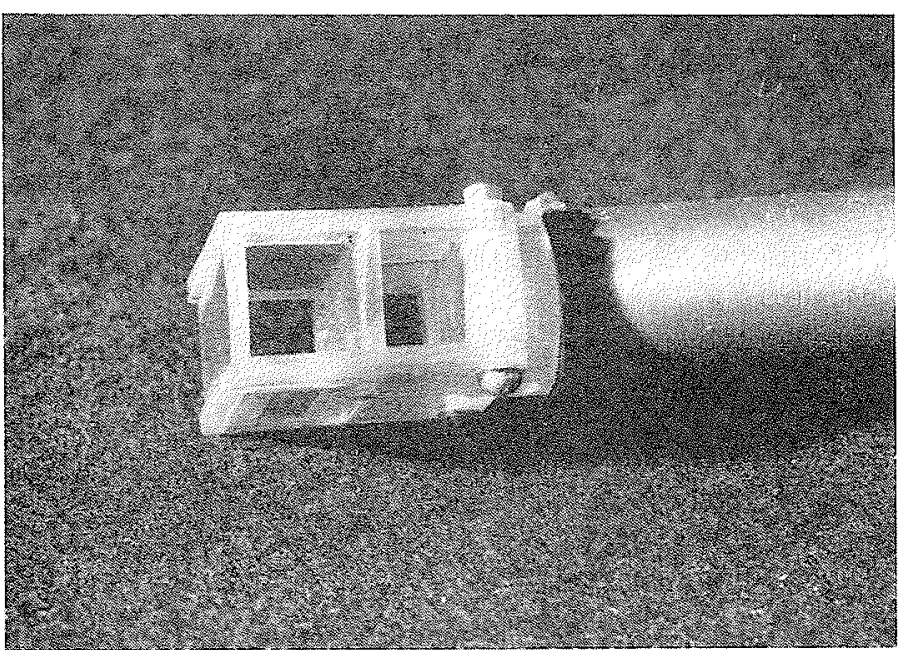

Fig. 5. SONDE $\mathrm{N}^{\circ} 2$.

tives. Nous avons alor's réalisé la sonde $\mathrm{n}^{\circ} 2$ (fig. 5) analogue à $\operatorname{la}^{\circ} 1$, mais de dimensions plus faibles $(20 \times 10 \times 10)$ et comportant deux joues en plexiglass limitant le champ dans le sens du gradient de concentration. La figure 4 montre alors une variation de $1 / 1.000$ de $R_{0}$ lorsque le centre de la sonde s'approche à $8 \mathrm{~mm}$ de la paroi.

b) Les dimensions de la sonde demeuraient cependant trop importantes. Un obstacle dans

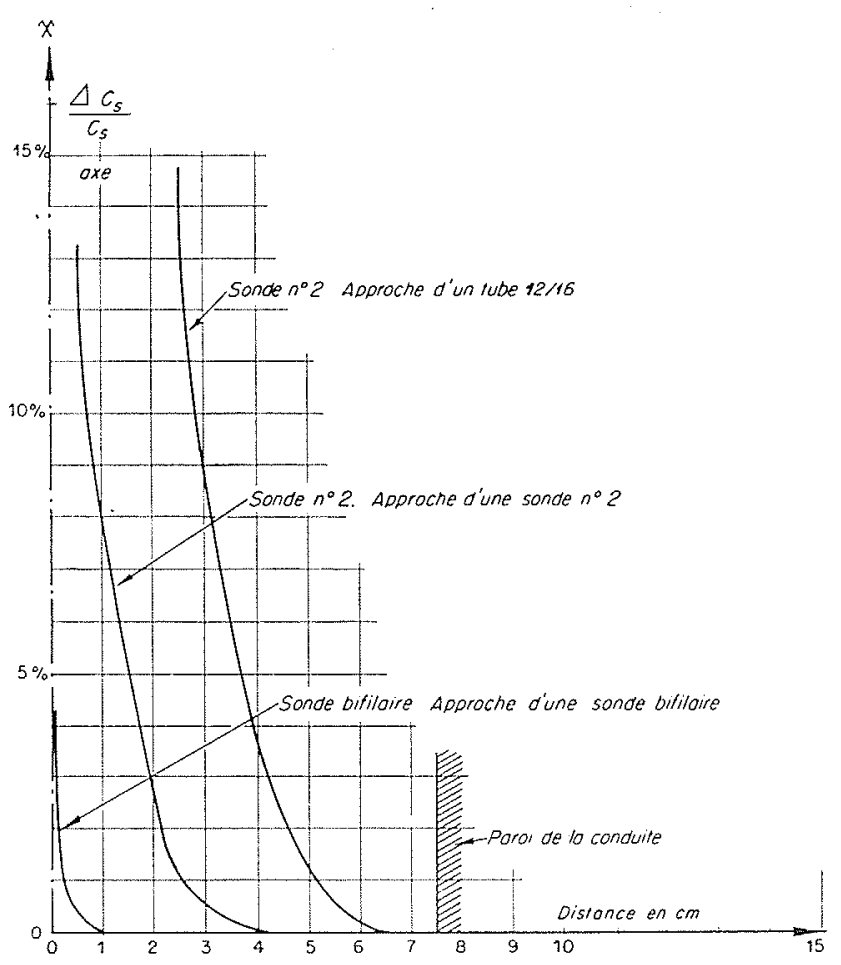

FIG. 6. l'écoulement crée en effet une courbure des lignes de courant; le rapport de densité de l'eau et de l'air étant d'environ 800 , les deux fluides ne suivent pas les mêmes trajectoires, et l'air s'accumule vers le centre de courbure. La mixture au voisinage de l'instrument de mesure risque donc d'être différente de celle qui s'écoule au mème point lorsque cet obstacle n'est pas présent. Pour mettre ce fait en ívidence, nous avons disposé une sonde-témoin fixe, au centre de la conduite; les conditions de débit liquide of gazeux restant invariables, nous introduisions par le second bossage à $90^{\circ}$, soit un tube schématisant le tube support, soil une sonde mobile idntique à la sonde témoin. La figure 6 montre les variations de la concentration indiquée par la sonde témoin; l'écart $\frac{\Delta \bar{c}_{s}}{\overline{c_{s}}}=14,3 \%$ entre les

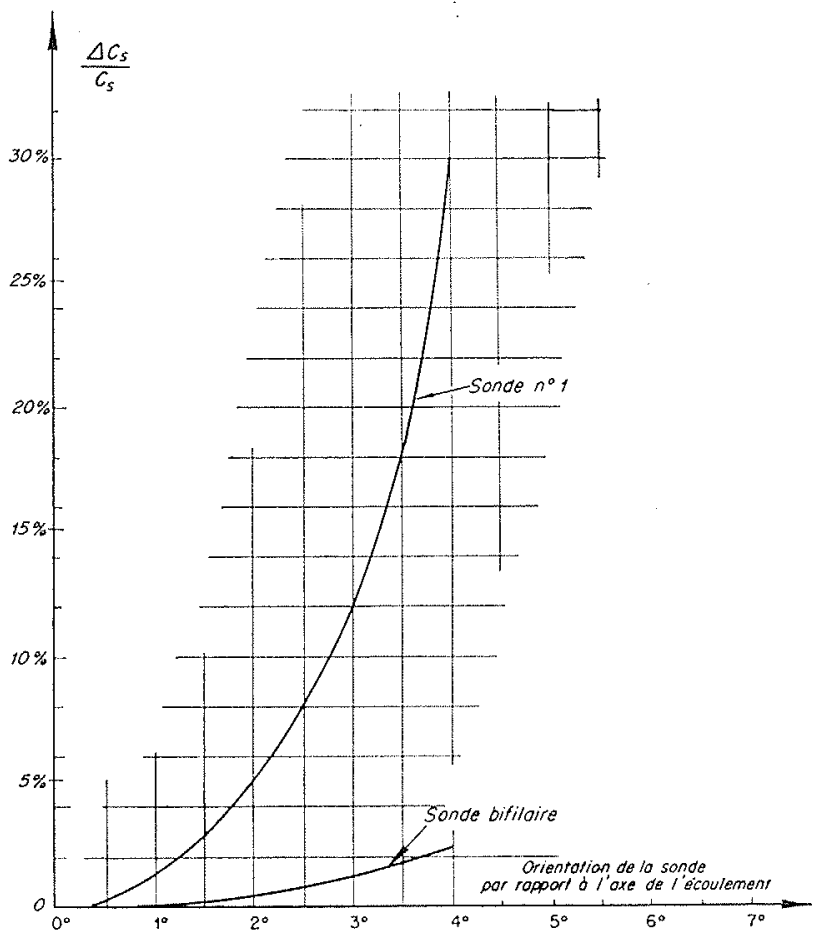

Fic. 7.

lectures lorsque l'obstacle est très éloigné et lorsqu'il est rapproché indique la perturbation considérable apportée à l'écoulement par la présence de l'obstacle mobile.

c) Enfin la figure 7 montre les variations de la concentration lue par la sonde en fonction de son inclinaison sur les lignes de courants. Une inclinaison de $4^{\circ}$ introduit une erreur $\frac{\Delta \overline{c_{s}}}{\overline{c_{s}}}=30 \%$. Il est donc nécessaire, avec une telle sonde, de procéder à un calage en direction très minutieux. 


\section{V. - Sonde bifilaire}

\section{1) Principe}

Devant ces difficultés, nous avons cherché à réaliser une sonde dont les dimensions soient petites vis-à-vis de celles des bulles gazeuses; le dispositif utilisé comporte deux fils fins émaillés, torsadés mutuellement et dénudés chacun sur la partie de leur surface qui n'est pas en contact avec l'autre fil. S'ils sont portés à un potentiel différent, le circuit électrique se ferme à travers

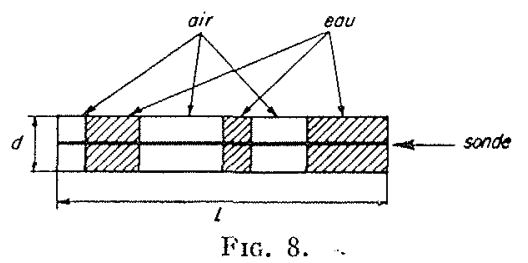

l'électrolyte et l'expérience montre que le diamètre utile $d$ du champ est de l'ordre du diamètre des fils. Supposons alors la sonde bifilaire, de longueur $l$, immergée dans un écoulement mixte, parallèlement aux lignes dejcourant (fig. 8). Soit un tube cylindrique de courant axé sur la sonde et de diamètre $d$; si d est petit devant le diamètre des bulles, celles-ci découpent à un instant donné, à l'intérieur du tube, un certain nom- bre de cylindres de longucur tolale $l_{1}$. A cet instant, dans le tube de courant :

$$
c_{8}=\frac{l_{1}}{l}
$$

L'écoulement moyen ctant supposé uniforme, la moyenne dans le temps do $c_{s}$ est la mòme en tout point de la sonde, donc :

$$
\bar{c}=\frac{l_{1}}{l}
$$

L'air étant isolant par rapport à l'eau, la résistance électrolytique est inversement proportionnele à la longueur de sonde baignée par l'eau :

D’où :

$$
\begin{aligned}
\mathrm{R}_{0} & =\frac{\mathrm{K}}{l} \\
\mathrm{R} & =\frac{\mathrm{K}}{l-l_{1}}
\end{aligned}
$$

$$
\overline{c_{s}}=\frac{\overline{\mathrm{R}-\mathrm{R}_{0}}}{\mathrm{R}}
$$

\section{2) Réalisation}

Le matériau adopté pour la construction de la sonde a été du fil de constantan émaillé de $0,3 \mathrm{~mm}$ de diamètre, qui a donné toute satisfac-

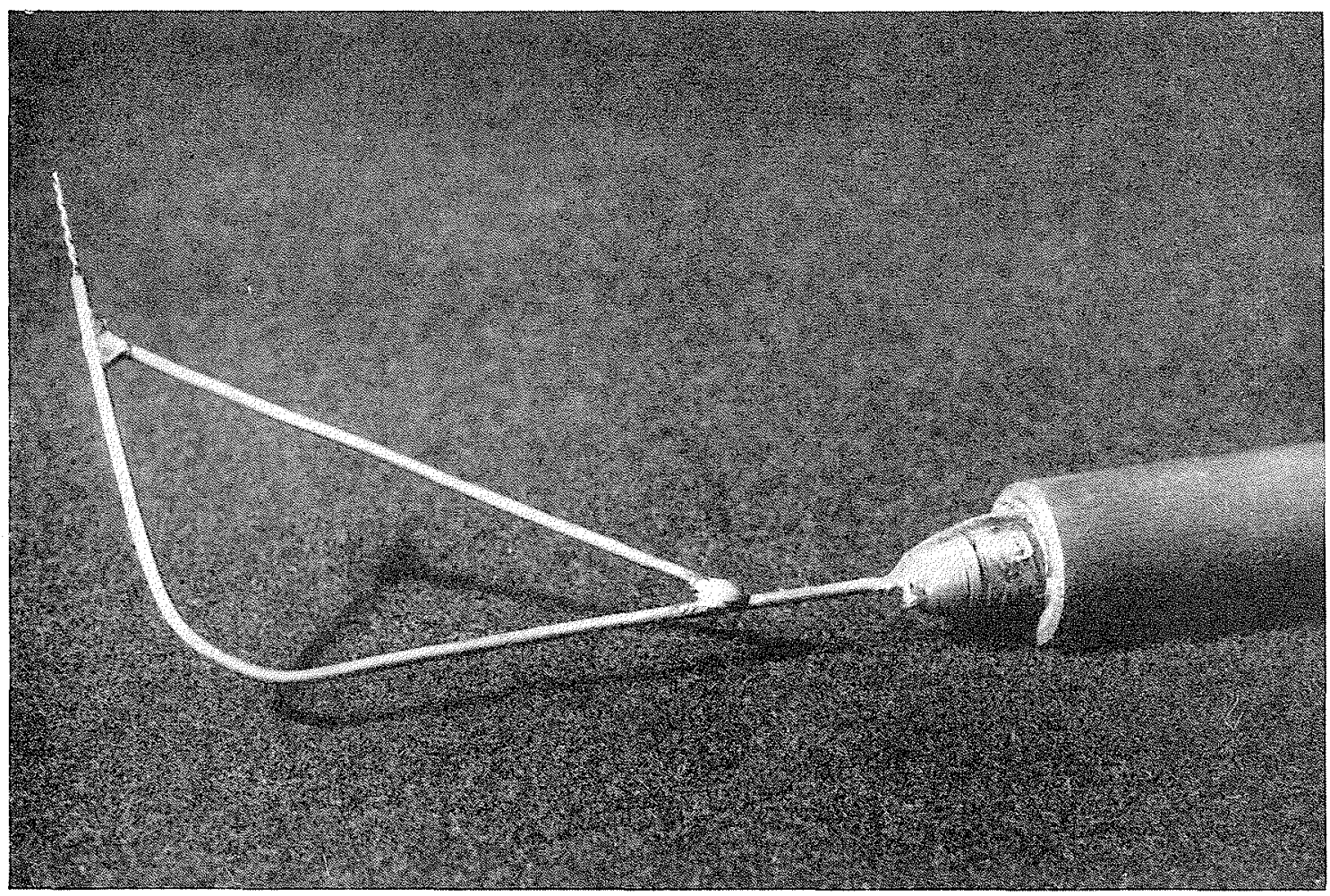




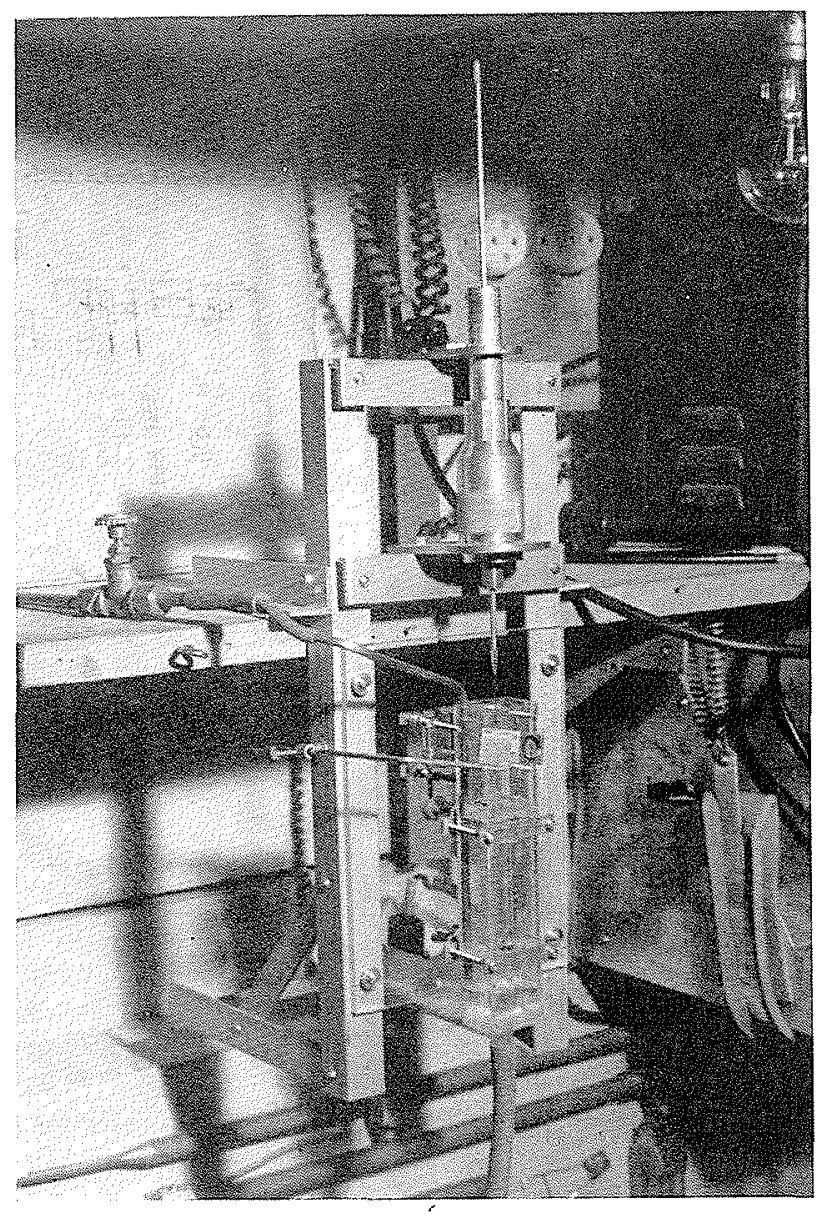

Fig. 10. - Dispositif D'Étalonnage statiguts.

tion au triple point de vue de l'invariabilité des caractéristiques électriques, de l'isolement et de la résistance mécanique.

Après divers tâtonnements, la sonde définitive (fig. 9) a été constituée par un tube de garde (diamètre extérieur $1,5 \mathrm{~mm}$ ) prolongé par un cône de raccordement en plexiglass d'où émergent à l'avant les deux fils torsadés, dénudés sur un centimètre de longueur. Les deux fils isolés se prolongent dans le tube support et se raccordent aux fils de liaison avec l'appareillage de mesure dans un boîtier étanche situé hors d'ean. Seul le tube de garde très fin se trouve donc immergé à l'aval des électrodes de mesure.

\section{VI. - Etalonnage de la sonde bifilaire}

Il était nécessaire de vérifier les hypothèses sur lesquelles repose l'établissement de la formule (5). D'autre part, nous désirions examiner si les inconvénients signalés à propos des sondes à plaques étaient éliminés.

\section{1) Première Méthode}

Cet essai, purement statique, visait à vérifier la validité de la formule fondamentate (4) et à étudier l'incidence des caractéristiques constructives de la sonde sur son comportement électrique.

Pour cela, une bulle d'air était mise en équilibre dans un tube $(\varnothing=4 \mathrm{~mm})$ paraffiné intérieurement et empli d'eau. La sonde à essayer était déplacée suivant l'axe du tube au moyen d'une vis micrométrique permettant d'apprécier le $1 / 100$ de $\mathrm{mm}$. Une circulation d'eau extérieure maintenait constante la température du tube (ig. 10).

La figure 11 montre la variation de $\frac{1}{R}$ en fonction des positions relatives de la bulle et de la sonde. Les deux parties à pente opposée correspondant à l'entrée et à la sortie de la sonde dans la bulle, la partie horizontale au déplacement de la sonde à travers la bulle. Un tel graphique confirme parfaitement la validité de la formule (4).

Grâce à ce dispositif, nous avons pu constater en outre que l'influence du champ des bouts était tout à fait négligeable.

\section{2) Seconde méthode.}

Après cet essai purement slatique, nous avons étudié le comportement de la sonde dans un courant de bules d'air traversant de l'eau au repos. L'installation comportait un tube de verre vertical de $12 \mathrm{~mm}$ de diamètre, empli d'eau jusqu'à un niveau $h_{0}$ lụ à l'aide d'un curseur à vernier sur une règle graduée. Un débit d'air constant était injecté à la base du tube à travers une aiguille hypodermique; les bulles produites avaient un diamètre approximatif de $5 \mathrm{~mm}$ et une vitesse ascensionnelle $\overline{v_{g}}$ à peu près constante sur toute la longueur du tube. Si $h_{1}$ représente le niveau de la surface libre en cours de souflage, le volume d'air contenu dans le tube de section $\Sigma$ était à un instant donné $\mathrm{\Sigma}\left(h_{1}-h_{0}\right)$. Ici, $\bar{Q}$ est nul, mais on a d'après (2) :

$$
\overline{\mathrm{Q}}_{t}=\overline{\mathrm{C}}_{\mathrm{s}} \bar{v}_{g} \Sigma
$$

$\overline{\mathrm{Q}}_{a}, \bar{v}_{g}$ et $\Sigma$ étant constants tout le long du tube, il en est de même de $\overline{\mathrm{C}}_{s}$; comme :

$$
\left.\Sigma \overline{\left(h_{1}\right.}-h_{0}\right)=\overline{Q_{a}} \frac{\overline{h_{1}}}{\overline{v_{g}}}
$$

on doit avoir :

$$
\overline{\mathrm{C}}_{s}=\frac{\overline{h_{1}}-h_{0}}{\overline{h_{1}}}=1-\frac{h_{0}}{\overline{h_{1}}}
$$




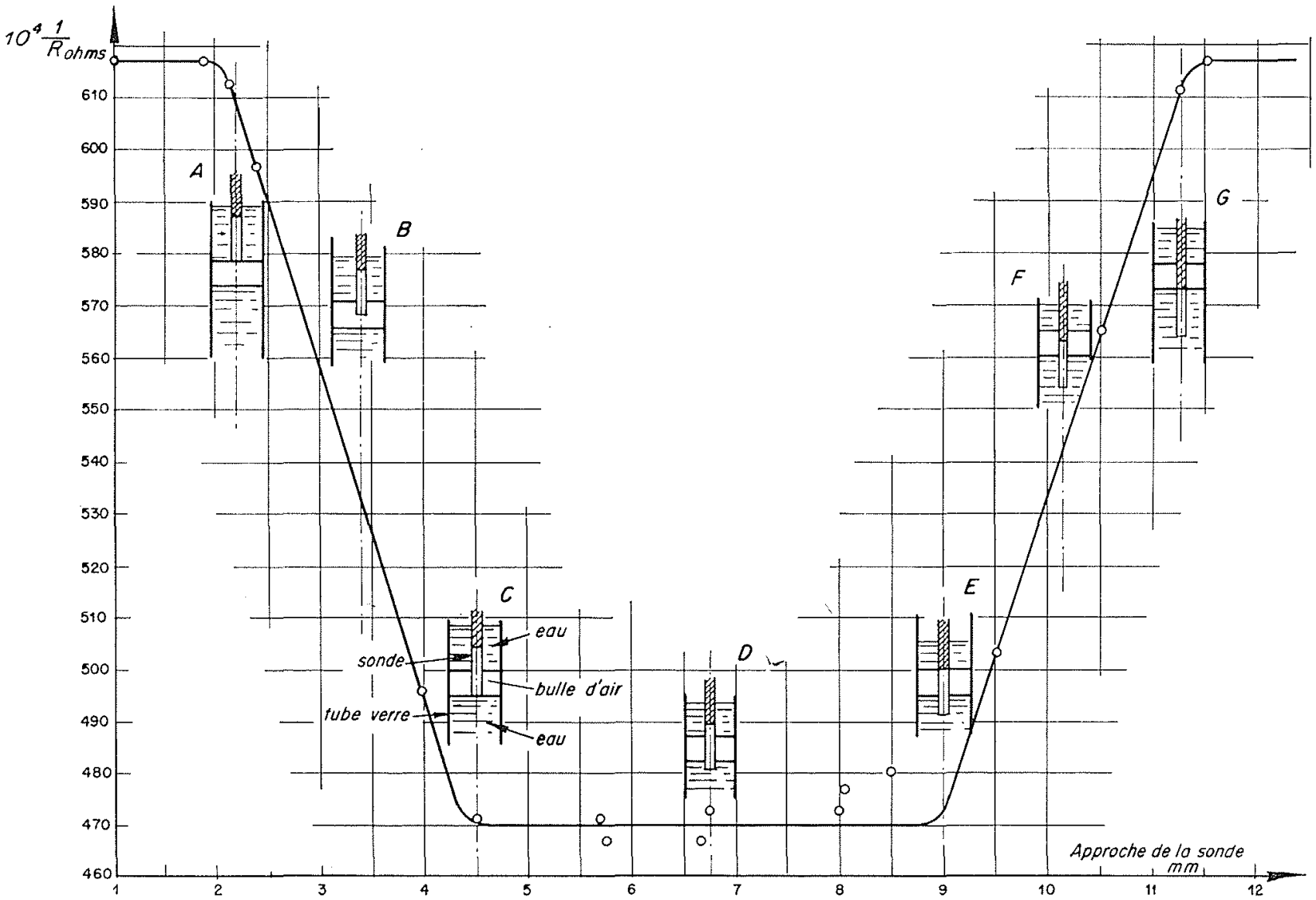

Fig. 11.

Le tableau II permet de comparer les valeurs mesurées de $\overline{\mathrm{C}}_{s}$ et de $1-\frac{h_{0}}{h_{1}}$.

'Tableau II

\begin{tabular}{|r|r|r|c|}
\hline $\begin{array}{c}\text { Essai } \\
n^{\circ}\end{array}$ & $1-\frac{h_{0}}{h_{1}}$ & \multicolumn{1}{|c|}{$\mathrm{C}_{s}$} & $\begin{array}{c}\text { Erreur relative } \\
\%\end{array}$ \\
\cline { 2 - 3 } & & & \\
\hline 1 & 3,65 & 3,58 & $-1,9$ \\
2 & 7,35 & 7,18 & $+0,47$ \\
3 & 12,60 & 12,90 & $-2,18$ \\
4 & 6,40 & 6,33 & $+2,18$ \\
5 & 6,70 & 6,60 & $-1,49$ \\
6 & 7,62 & 7,52 & $-1,33$ \\
7 & 4,58 & 4,50 & $-1,74$ \\
8 & 2,19 & 2,20 & $+0,45$ \\
9 & 11,05 & 9,95 & $-1,00$ \\
10 & 15,70 & 16,14 & $+2,80$ \\
\hline
\end{tabular}

Les écarts sont faibles; on peut d'ailleurs préciser ce point; la principale erreur provient de la mesure de $\bar{h}_{1}$ car le niveau libre fluctue d'autant plus que le débit d'air introduit est plus élevé ct la prise de la moyenne à l'wil est sujette à caution. On a par différenciation de (6) :

$$
\frac{d \overline{\mathrm{C}}_{s}}{\overline{\mathrm{C}}_{s}}=\frac{h_{0}}{\overline{h_{1}}-h_{0}} \frac{d \bar{h}_{1}}{\widetilde{h}_{1}}
$$

On en déduit pour les débits extrêmes :

Expérience $1: d \vec{h}_{1}=0,47 \mathrm{~mm}$

Expérience $10: d \bar{h}_{1}=3,8 \mathrm{~mm}$

Ces valeurs paraissent demeurer dans le domaine des erreurs expérimentales possibles.

\section{3) Trorsnème méthode.}

Le fonctionnement de la sonde paraissant conforme aux prévisions dans les deux cas particuliers précédents, il restait à contrôler qu'il en était encore de même pour un écoulement de 
mixture. Les expériences dans la conduite d'essai décrite plus haut ont montré les points suivants :

a) la variation de $R_{0}$ n'est que de 5/1.000 lorsque le tube de garde vient au contact de la conduite; l'axe de la sonde est alors à $0,75 \mathrm{~mm}$ de la paroi (voir fig. 4). Ce fait justifie notre affirmation concernant les dimensions utiles au champ électrique; il montre que la sonde permet la mesure de $\bar{c}_{8}$ au voisinage du fond d'un canal.

b) suivant la méthode précédente, l'approche d'une sonde mobile et d'une sonde témoin, jusqu'au contact des tubes de garde, produit une variation $\frac{\Delta \bar{c}_{s}}{\overline{c_{s}}}=4,3 \%$ indiquant une faible perturbation de l'écoulement (voir fig. 6);

c) enfin, dans un écoulement mixte, uniforme (ou à faible gradient de concentration), l'indication de la sonde est peu sensible à une erreur accidentelle d'orientation; la figure 7 indique une variation $\frac{\Delta \bar{c}_{s}}{\overline{c_{s}}}$ de $2,2 \%$ pour une inclinaison de $4^{\circ}$ de la sonde sur les lignes de courant.

En dépit de toutes ces améliorations, la valeur moyenne lue $\overline{\mathrm{C}}_{s}$ demeurait constamment inférieure à $C_{\mathrm{T}}$. Ce fait pourrait être attribué :

- à une répartition non uniforme de $\bar{v}_{c}$,

-.- à une vitesse de glissement $\bar{v}_{y}$ non négligeable.

En effet, dans ce cas, en supposant $\bar{v}_{g}$ constant dans toute la section $\mathrm{S}$ et en posant :

$$
\overline{\mathrm{V}}_{e}=\frac{1}{\mathrm{\Sigma}} \iint_{\mathrm{\Sigma}} \bar{v}_{\mathrm{e}} d \sigma
$$

on obtient, par addition de (1) et (2) :

$$
\overline{\mathrm{Q}}_{e}+\overline{\mathrm{Q}}_{a}=\Sigma\left(\overline{\mathrm{V}}_{e}+\bar{v}_{y} \overline{\mathrm{Q}}_{\mathrm{s}}\right)
$$

d'où :

$$
\mathrm{C}_{\mathrm{IV}}=\overline{\mathrm{C}}_{s}\left[1+\frac{1-\overline{\mathrm{C}}_{s}}{\mathrm{~V}_{c} / v_{g}+\mathrm{C}_{s}}\right]>\overline{\mathrm{C}}_{s}
$$

Pour conclure, il ćlait indispensable de combiner la mesure des concentrations spatiales et celle des vitesses locales.

\section{VII. - Principe de la mesure des vitesses}

\section{1) Appareil utilist́.}

Cette mesure a été effectuce au moyen d'une prise dynamique constituée par un élément de tube identique au tube de garde de la sonde électrique. Dans la section de mesure horizontale, les filets liquides sont parallèles, done la pression statique est constante. Il suffit, pour la mesurer, d'une prise de pression à la paroi; prise dynamique et prise statique sont reliées à un manomètre différentiel à eau qui mesure une dénivellation H. Un jeu de robinets à trois voies permet une purge aisée à l'aide d'eau sous pression; le courant de purge étant établi dans le sens manomètre-prise, de l'eau s'écoule constamment par la prise entre la fin de la purge et l'instant de la mesure, empêchanl ainsi l'air de pénétrer dans les tuyaux, ce qui risquerait de fausser les lectures.

\section{2) Indications de L'Appareil.}

La présente du tube de PrTot introduit des courbure des lignes de courant; imaginons pour un instant que les bulles d'air suivent les mêmes trajectoires que les filets fluides; la valeur de la concentration spatiale $c_{s}$ au voisinage de la pries demeurera identique à sa valeur lorsque l'obstacle est absent. Soit un petit volume $d V$ situé devant le tube; sur une période de temps $\mathrm{T}$, longue, ce volume est rempli d'eau pendant le temps global $t_{1}$ et la prise dynamique subit une surpression :

$$
i_{e} \frac{v_{c}^{2}}{2 g}
$$

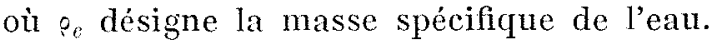

Pendant le temps $t_{2}=\mathbf{T}-t_{1}$, le volume $d V$ est rempli d'air et la prise subit la surpression :

$$
\rho_{a} \frac{v_{a}^{2}}{2 g}
$$

où of désigne la masse spécifique de l'air. Etant donné la grande constante de temps du tube de diamètre $1,5 \mathrm{~mm}$ et des tuyauteries de liaison, le manomètre différentiel indique pratiquement la moyenne dans le temps des surpressions instantanées. Si les fluctuations de $v_{e}$ et de $v_{a}$ sont laibles :

$\mathrm{H}=\rho_{e} \frac{\bar{v}_{e}^{2}}{2 g} \frac{t_{1}}{\mathrm{~T}}+\rho_{a} \frac{\bar{v}_{a^{2}}}{2 g} \frac{t_{2}}{\mathrm{~T}}=\rho_{c}\left(1-\bar{c}_{s}\right) \frac{\bar{v}_{b}^{2}}{2 g}+\rho_{a} \bar{c}_{s} \frac{\bar{v}_{a}{ }^{2}}{2 g}$ 
3) Conframer de captation.

En réalité, nous avons déjà signalé que les bulles d'air se déplacent vers le centre de courbure des filets fiuides; à l'instar de ce qui a déjà été fait pour les suspensions solides (4), introduisons un coefficient de captation $\gamma$ qui peut être défini comme le rapport de la valeur de la concentration spatiale en air devant la prise à sa valeur au même point lorsque l'obstacle est absent $(0 \leqslant \gamma \leqslant 1)$. Les valeurs des vitesses étant peu modifiées, on a :

$$
\mathrm{H}=\rho_{c}\left(1-\gamma \bar{c}_{s}\right) \frac{\bar{v}_{e}^{2}}{2 g}+\tau_{a} \gamma \bar{c}_{s} \frac{\bar{v}_{a}^{2}}{2 g}
$$

Il est facile de voir que :

$$
\gamma=f\left(d, \mathrm{D}, v_{\ell}, \rho_{e}, \varrho_{c}, g, \bar{c}_{s}\right)
$$

d désignant le diamètre des bulles, D celui du tube de Prtor, $\mu_{o}$ la viscosité de l'eau; $\rho_{a}$ est très faible devant $?_{e}$ et n'intervient pratiquement pas.

Un calcul d'analyse dimensionnel conduit alors au groupement suivant des variables :

$$
\gamma=\gamma\left(\frac{d}{\mathrm{D}}, \frac{v_{e} d}{v_{e}}, \frac{v_{e}^{2}}{g \mathrm{D}}, \overline{c_{s}}\right)
$$

où $y_{c}=\frac{\mu_{e}}{\rho_{e}}$.

$\frac{v_{e}^{2}}{g \mathrm{D}}$ mesure le rapport de l'accélération locale due à l'obstacle $\frac{D_{e}^{2}}{\mathrm{D}}$ à l'intensité de la pesanteur; dans nos expériences, $v_{c}$ est grand et $D$ petit, l'accélération locale l'emporte, la pesanteur n'intervient plus et le terme $\frac{v_{e}{ }^{2}}{g \mathrm{D}}$ disparaît.

Le nombre de Reynolds $\frac{v_{e} d}{v_{e}}$ influe par la valeur du coefficient de traînée $C_{x}$ qui détermine la résistance rencontrée par la bulle dans son mouvement relatif; or, si $v_{c}=500 \mathrm{~cm} / \mathrm{s}, d=0,2 \mathrm{~cm}$, $\nu_{c}=0,01 \mathrm{~cm}^{2} / \mathrm{s}, \frac{v_{e} d}{y_{e}}=10^{4}$ et dans cette gamme de nombre de Reynolds $C_{v}$ est pratiquement constant. Le terme $\frac{\nu_{c} d}{y_{e}}$ disparait donc lui aussi et on aboutit à la relation simple approchée :

$$
\gamma=l\left(\frac{d,}{\mathrm{D}} c_{s}\right)
$$

(4) Brux, Carow et Vasseun. Introduction a l'étude de la méeanique des suspensions. G.R.A., Rapport technique $n^{0}$ 15, Paris, 1945.
On pent tenter de prévoir qualitativement le sens de variation en fonction de $\frac{d}{\mathrm{D}}$ et de $\overline{c_{s}}$.

a) Pour $\overline{c_{s}}=0$ et $\overline{c_{s}}=1, \gamma=1$.

b) Lorsque $d$ augmente, D restant constant, les forces d'inertic pioportionnelles à $d^{3}$ croissent plus vite que la résistance hydrodynamique qui est proportionnelle à une puissance de $d$ comprise entre 1 et 2 . Il semble done que $\%$ doive diminuer lorsque $\frac{d}{\mathrm{D}}$ augmente et que les courbes reprísentatires de y aient l'allure représentée figure 12 .

L'observation visuelle nous a montré que, pour la convection forcée réalisée dans la conduite, les bulles d'air sont de dimensions impor-

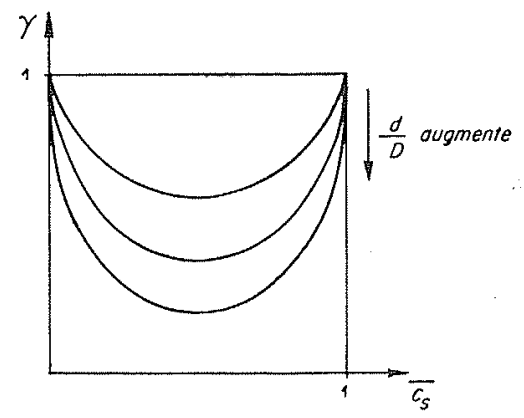

Fic. 12

tantes devant D; d'autre part, nous réalisons des émulsions dont la concentration $\overline{\mathrm{C}_{\beta}}$ est voisine de $20 \%$; on doit donc commettre une erreur faible en prenant $\gamma=0$; comme $\frac{\rho_{e}}{o_{a}} \# 800$, la surpression due à l'air demeure très petite. Finalement :

$$
H \# \varphi_{c} \frac{\bar{v}_{e}^{2}}{2 g}
$$

Or, indépendamment de toute hypothèse sur la distribution des vitesses dans la section de mesure et sur la valeur de la vitesse de glissement, on a l'égalité :

$$
\overline{Q_{e}}=\iint_{\mathrm{S}}\left(1-\bar{c}_{s}\right) \bar{v}_{e} d \sigma
$$

Si le fonctionnement de lat sonde bifilaire et du tube de PrTot est correct, leurs indications doivent vérifier eette relation où le débit liquide est mesuré au moyen du déversoir. 

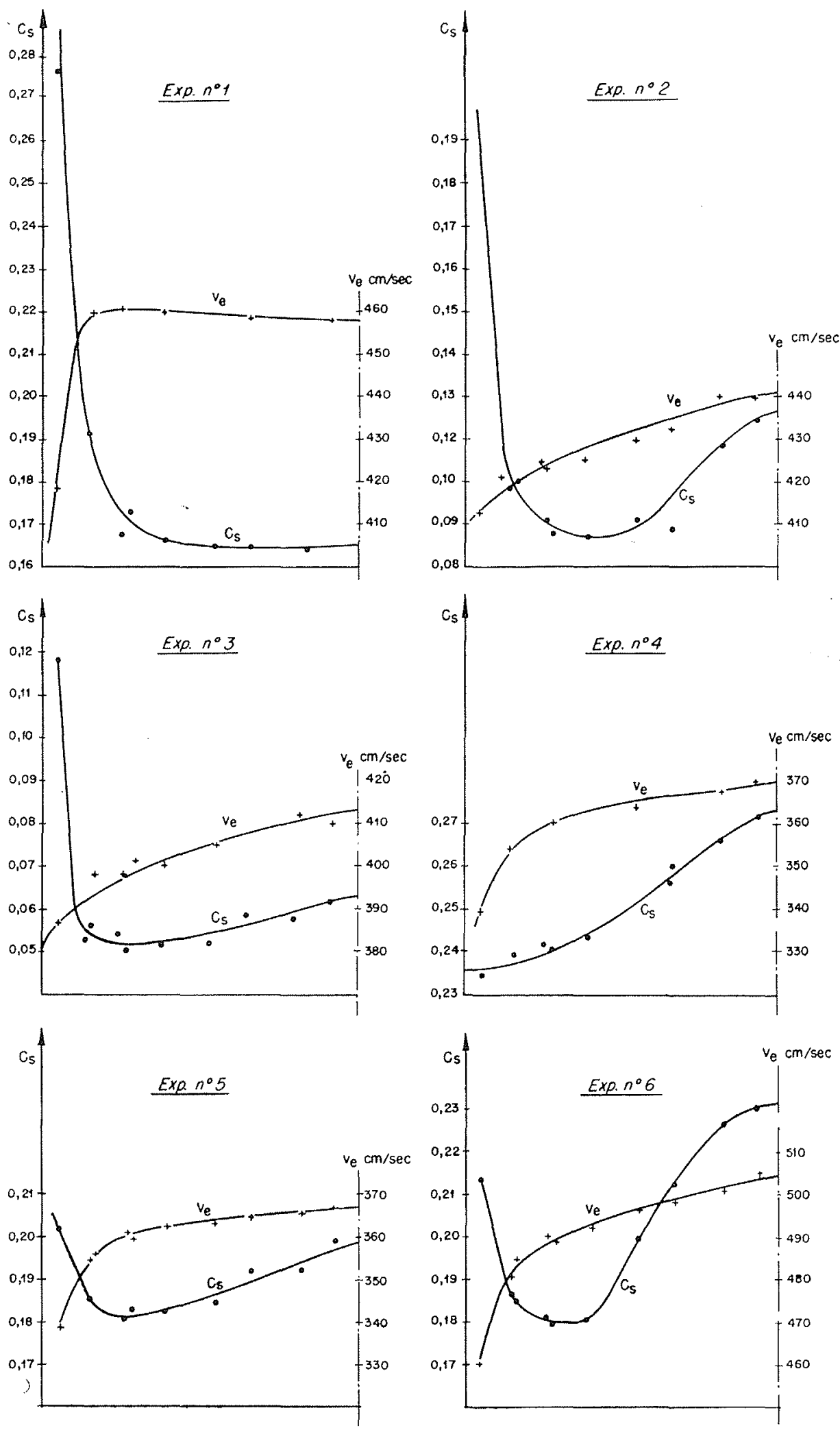

Fiti. 13 


\section{VIII. - Vérification expérimentale} du fonctionnement correct des sondes

Nous avons utilisé une sonde électrique et une prise dynamique montées à un centimètre de distance sur un tube commun $(\varnothing=1,5 \mathrm{~mm})$; par translation de ce tube, nous pouvions explorer deux diamètres de la section de mesure. Le tableau III et la figure 13 résument les résultats de ces expériences. On constate que l'accord entre les valeurs mesurées et calculées de $Q_{e}$ est réalisé avec une erreur inférieure à $1 \%$, ceci pour de larges variations de débit d'air injecté.

Il faut remarquer que la valeur de $\overline{\mathrm{Q}}_{1}$ n'a pas été utilisée dans ce processus de vérification : sa mesure permet cependant de se faire une idée de l'ordre de grandeur de $v_{g}$ supposé constant dans toute la section $\Sigma$. En effet, on tire de (7):

$$
\bar{v}_{g}=\frac{\overline{\mathrm{Q}}_{c}+\overline{\mathrm{Q}}_{a}-\overline{\Sigma \mathrm{V}}_{e}}{\Sigma \overline{\mathrm{C}}_{s}}
$$

On notera dans le tableau III les fortes valeurs obtenues pour $\bar{v}_{q}$. On s'éloigne en réalité beaucoup des conditions de Mryagr et chaque bulle profite ici du sillage de celles qui la précèdent. Elle se trouve donc beaucoup moins freinée que lorsqu'elle est isolée. De plus, la figure 12 montre que, lorsque $\overline{\mathrm{C}_{s}}$ est faible, l'air est bien distribué dans toute la section, sauf au voisinage des parois où la valeur de $\bar{c}_{s}$ augmente beaucoup. Lorsque $\overline{\mathrm{C}_{s}}$ croît, une partie de l'air se rassemble dans l'axe de la conduite, en formant une sorte de cheminée où son mouvement doit être fort peu freiné. Enfin il est possible que l'eflet accélérateur du convergent soit encore sensible dans la section de mesure.
TABLEAU III

\begin{tabular}{|c|c|c|c|c|c|c|}
\hline $\begin{array}{c}\text { Expérience } \\
n^{\circ}\end{array}$ & 1 & 2 & 3 & 4 & 5 & 6 \\
\hline$\overline{\mathrm{Q}}_{b} \mathrm{l} / \mathrm{s}$ & 20,1 & 12,0 & 7,7 & 28,2 & 19,5 & 25 \\
\hline$\overline{Q_{e}} l / s$ & 63,2 & 65,4 & 65,6 & 47,6 & 50,6 & 68,8 \\
\hline $\int_{\Sigma}\left(1-\bar{c}_{s}\right) \bar{v}_{c} d \sigma$ & 63,2 & 65,8 & 65,9 & 47,5 & 51,1 & 68,85 \\
\hline$\overline{\mathrm{V}}_{e} \mathrm{~cm} / \mathrm{s}$ & 445 & 424 & 400 & 356 & 349 & 481 \\
\hline$\overline{\mathrm{C}_{s}}$ & 0,190 & 0,122 & 0,066 & 0,245 & 0,186 & 0,19 \\
\hline $\bar{v}_{q} \mathrm{~cm} / \mathrm{s}$ & 133 & 113 & 224 & 297 & 246 & 219 \\
\hline
\end{tabular}

\section{IX. - Conclusion}

Des expériences précédentes, on peut conclure que la sonde bifilaire, telle qu'elle a été décrite, permet une mesure correcte des concentrations spatiales moyennes en chaque point dans un courant mixte d'air et d'eau où l'air est présent sous la forme de bulles et où la vitesse est de l'ordre de quelques mètres par seconde. Dans le même domaine, une prise dynamique permet une mesure commode de la vitesse, moyennant une estimation préalable de la valcur du coefficient de captation.

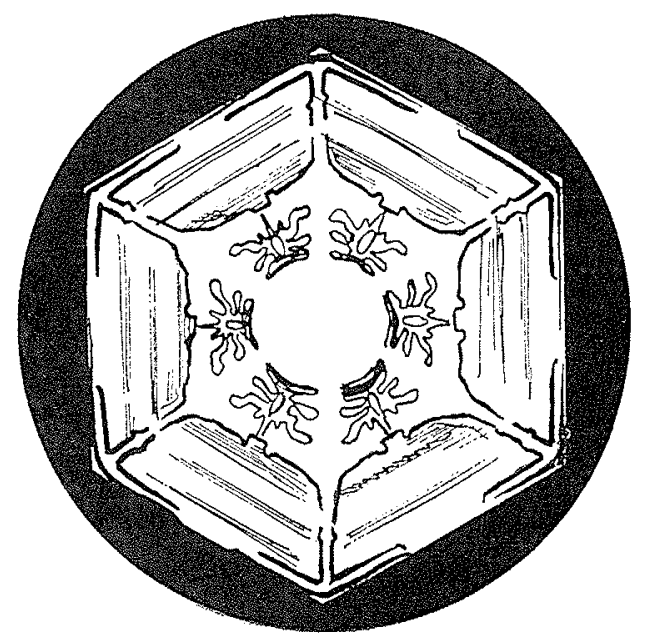

